\title{
An Analysis Method for Heating Network Leaks Monitoring
}

\author{
Ilia TRIAPITCIN ${ }^{\mathrm{a}}$ and Ajantha DAHANAYAKE ${ }^{\mathrm{a}}$ \\ ${ }^{a}$ Lappeenranta-Lahti University of Technology Lappeenranta, \\ Finland, Yliopistonkatu 34 \\ ilia.triapitcin@student.lut.fi, \\ ajantha.dahanayake@lut.fi
}

\begin{abstract}
Modern district heating (DH) systems are complex engineering structures that play an essential role in large city infrastructures. DH networks have many sensors, nodes, and methods for monitoring the status of the DH network. Sensing, processing, analytical actuation (SPA) of incoming information handled by the SPA semantic Computing method can be applied to similar problems. The SPA Semantic Computing method searches for correlations between the sets of incoming data and to identify the correct scenario to respond to events. This article explores the integration of SPA functions to analyze multivariate sensing data, including data from multivariable sensors and infrared images, for creating a monitoring system for DH networks. The focus is to assess whether the SPA approach is a suitable candidate to use to monitor the emergency events of the DH network. Specific target data for the assessment are [1] multi-parameter DH network sensor data, such as water temperature, sweat rate, energy delivered, etc., and [2] infrared image data from a camera mounted on the unmanned aerial vehicle (UAV) for monitoring the location of the underground DH network leaks. A multivariate computational model, a mathematical model of meaning (MMM), and a spatial image filtering method are proposed for integrating SPA semantic computing for emergency leak detection in DH networks.
\end{abstract}

Keywords. district heating, geographical information system, sensor data, differential computing, semantic computing.

\section{Introduction}

District heating $(\mathrm{DH})$ or remote heating is a method for distributing heat generated in a heat center (HC) through insulated pipes for individual and commercial heating needs such as space heating and water heating. Unfortunately, water leaks from the district heating network are disastrous during Nordic winters. Therefore, finding leaks as soon as possible is necessary to reduce any unwanted accidents.

Modern DH networks are complex engineering structures monitored by hundreds of sensors in real-time. In addition, there are many different analysis methods for localizing accidents in the DH system [1-3]. Therefore, many highly qualified staffs are engaged to analyze all the incoming information in the shortest possible time. This study explores the possibility of introducing sensing, processing, analyzing, and actuation (SPA) semantic computing method [4] to automate the DH network states monitoring and leak detection. As a result, it helps reduce the number of company staff needed for constant manual monitoring and, in turn, increases the response time during an emergency.

This article presents how the industry can use the SPA semantic computing method can be used to analyze the DH network's multivariate sensor data and thermal images 
around the surface of heating water pipelines for monitoring the system. This research suggests that integrating multimedia data and real-time sensor data analysis improves DH network analysis, facilitating emergency activity monitoring. Specific target data of the DH network are:

1. DH network sensor data, such as the incoming and outgoing water temperature, the movement of the incoming and outgoing flow, date of DH network construction, sensor's alarm data, water pressure inside tubes, and

2. the image data in the infrared range was obtained using a camera installed on the unmanned aerial vehicle (UAV).

The objectives of this study are to explore whether it is possible:

1. to analyze and generalize the spatial dynamics of the thermal data of the soil surface in the pipe-laying zone of the DH network, and

2. to integrate the semantic sequencing function and the spatial dynamics of the DH network data to assess the physical state of the DH network.

This research approach bases on the design science framework [4]. This research follows the design science research guidelines developed by Hevner et al. [4] for solving a relevant problem of the research field by creating an artifact to solve the problem while using the existing body of knowledge to arrive at an innovative solution. Finally, the artifact is validated for its relevance for the application domain. It extends the current scientific knowledge base with the new knowledge formulated for problem-solving in the environment of the research field. This process is illustrated in figure 1.

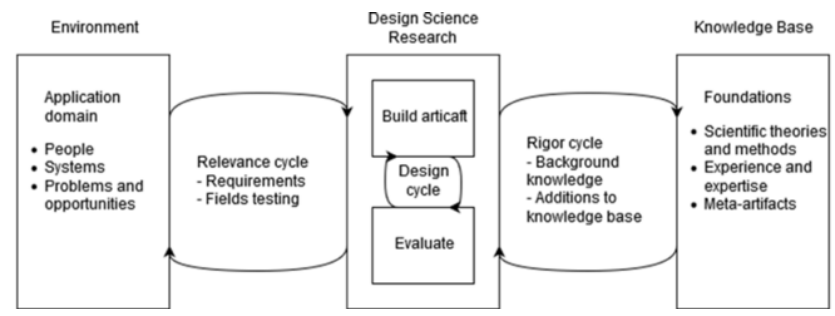

Figure 1. Design Science Research development life cycle.

The knowledge used from the body of knowledge is the SPA semantic computing concepts and method and the image processing and filtering methods. This knowledge foundation is used to explore an innovative solution for the DH network leak monitoring approach.

This article presents an overview of the five-dimensional monitoring and data analysis system for DH networks, and an overview of the system concept is provided and presented in Section 2. The method of the proposed detection, processing, and actuation functions in real-time is described in Section 3 while evaluating the proposed functions in Section 4. Finally, section 5 gives the conclusions together with further research directions. 


\section{Overview of the Theoretical Foundation}

In Y. Kiyoki at el. [5], the "5D World Map System" framework is a multivisualized and flexible information retrieval system used for environmental analysis and semantic computation. This framework defines the system as made up of five dimensions. The first dimension is the temporal dimension, then the second, third and fourth are spatial dimensions. The last one is the semantic dimension reflecting a large-scale and multidimensional semantic space based on the associative semantic computing system. The semantic dimension stores data from resources to correlate temporal and spatial areas. It implements the 5D world map to dynamically build Spatio-temporal and multiple semantic representations for diverse media resources.

The parameters of the DH network are treated as the fifth dimension in this article. This fifth dimension is depicted as a multi-dimensional space based on a mathematical model of meaning (MMM). MMM uses a semantic associative search approach for defining the concepts of "semantics" and "impressions" based on the "context" of infrared image tools. Figure 1 depicts the systems architecture for monitoring the condition of DH networks based on the design described in Y. Kiyoki et al. [5][6].

The DH network condition monitoring system is explored based on the "Sensing, Processing and Analytical Actuation" concept (SPA) [5][7][8]. "SPA" is efficient and beneficial in defining environmental phenomena in real space for real data tools. They map them to cyber-physical space to build analytical and semantic computation and visualize the analytically computed findings to the real space to convey environmental phenomena with causalities. In this study, the SPA definition is extended to semantic computation (see Figure 2). This system tries to reproduce human steps to handle the DH system's monitoring conditions and automate the monitoring process.
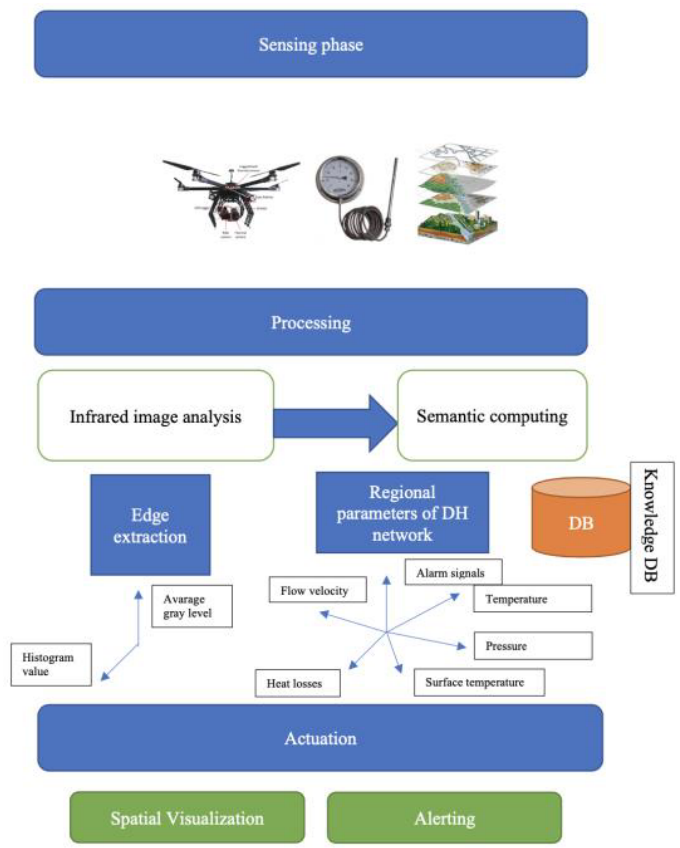

Figure 2. Concept of the system. 


\section{District Heating Measurements Analysis}

Semantic computing is based on semantic in terms of context, meaning, or intention. "SPA" is a foundational framework for implementing an environmental system of three essential functions, "Sensing, Processing and Analytical Actuation," to develop CyberPhysical integration and industrial engineering information systems. SPA helps define industrial phenomena in a physical space as real data tools, mapping them to cyber-space to build analytical and semantic computation, and visualize the analytically computed findings to the real space to express industrial phenomena with causalities [5]. Collected sensing data make the analysis space for finding correlations between different values. There are two other analysis spaces created in this research. The first space contains DH measurements from digital sensors inside the DH network. The second space is from digital thermal images collected by UAV. Sensing data and analysis spaces and are explained below.

\subsection{Multi-Parameter Sensing Data}

The DH network's stability is controlled by many parameters and is critical for the stability of the infrastructure. The multi-dimensional space for expressing the $\mathrm{DH}$ system's parameters for monitoring the DH network is shown in Figure $3[9,10]$.

- Air temperature axis: shows outside air temperature.

- Sensor's identification axis: is a unique number of a sensor in the DH network.

- Water temperature axis: shows the temperature of the water inside the DH network.

- Alarm indicator: shows emergency in the DH network branch

- The pressure inside tubes: shows water pressure inside the DH network pipe

- Flow velocity: parameters of the pressure loss of the water

- Customer needs: the amount of heat consumed by customers

- Building date: date is when the DH network or branch was built or renovated

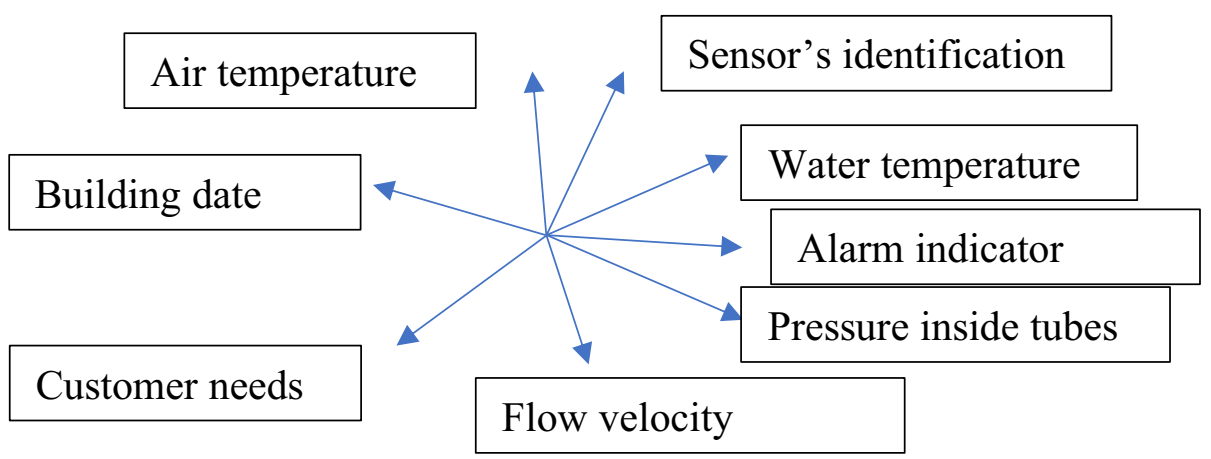

Figure 3. The multi-dimensional DH quality parameters.

The correlation between outside air temperature and input/output water temperature is presented in Figure 4. Also, the green line is the correlation between outside and inside temperature. 


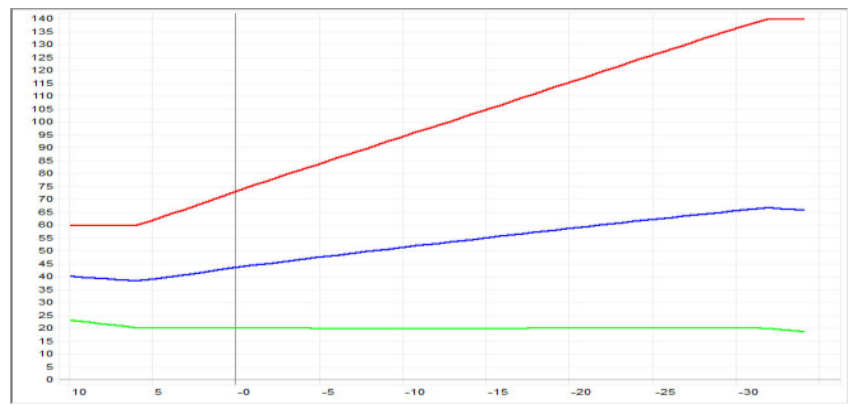

Figure 4. The temperature graph show correlation

between output water temperature (ordinate) and air temperature (abscissa): the red line is the input temperature of water; the blue line is output temperature of water; the green line is air temperature inside a customer building;[11].

The list of parameters is created at the design and modeling stage before constructing the industrial facility. However, in reality, the $\mathrm{DH}$ network is monitored by a lot of parameters. Therefore, a data vector is created of multiple DH parameters. The data vector contains a value of air temperature outside, inside customer's building, sensor's unique identification, alarm indicator, the water temperature on input and output of a pipe, the pressure inside a pipe, the velocity of the flow, total customer needs in power, building or renovation date. The data vector structure is presented in Table 1 . These data are collected in real-time and stored in a database.

Table 1. Data vector of DH parameters

\begin{tabular}{|c|c|c|c|c|c|}
\hline Sensor Id & $\begin{array}{c}\text { Temperature } \\
,{ }^{\circ} \mathrm{C}\end{array}$ & $\begin{array}{c}\text { Pressure, } \\
\text { MPa } \\
\left(10^{6} \frac{N}{m^{3}}\right) \\
\end{array}$ & $\ldots$ & $\begin{array}{c}\text { Velocity, } \\
m^{3} / h\end{array}$ & Needs, $G W t$ \\
\hline$V_{l 1}$ & $V_{i 1}$ & $V_{j 1}$ & $\ldots$ & $V_{k 1}$ & $V_{m 1}$ \\
\hline$V_{l 1}$ & $V_{i 2}$ & $V_{j 2}$ & & $V_{k 2}$ & $V_{m 2}$ \\
\hline$V_{l 1}$ & $V_{i k} \quad \cdots$ & $V_{j k} \quad \cdots$ & $\ldots$ & $V_{k n}$ & $V_{m n}$ \\
\hline
\end{tabular}

\subsection{Spatial District Heating Network Data}

Information about spatial DH networks is stored in the geographical information system. This data includes the spatial position of objects and all physical and technical parameters of the entities. The DH network example is shown in Figure 5.

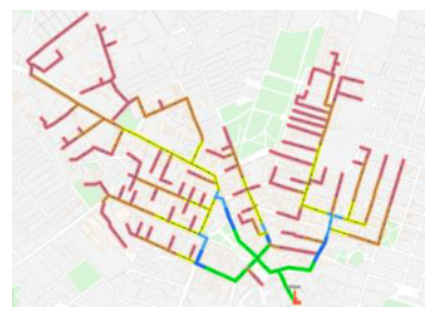

Figure 5. Spatial presentation of the DH network [11]. 


\subsection{Infrared Images}

The second space is for sensing images. UAV drone collects thermal images of the surface above the DH network. The collected images are handled during the preprocessing phase. This phase aims to find thermal anomalies on the soil surface associated with the DH network heat losses and water leaks.

\subsubsection{Image Preprocessing}

The original thermogram contains the absolute temperature values encoded in RGB palettes. It means that color code $\# 00000$ from the RGB palette corresponds to $0{ }^{\circ} \mathrm{K}$ or $273.16^{\circ} \mathrm{C}$. In reality, the thermogram of the Earth's surface in a settlement contains a temperature difference in the range of several tens of degrees. The minimum temperature value should be set arbitrarily to improve the visualization of gradients. The criterion for choosing a color is the distance from the opposite value. The optimal choice is blue (color code $\# 0000 \mathrm{FF}$ in the RGB palette) for the coldest area and red for the hottest site (color code \#FF0000 in the RGB palette). The original thermogram as a result of applying the filter is shown in Figure 6.
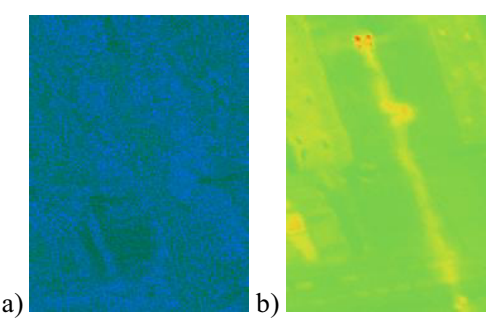

Figure 6. Selection of warm and cold areas: a) the original image; b) with the use of a filter; [11].

The feature extraction process from thermal images is based on the contrast enhancement of the image method proposed by Chiwu Bu et al. [12]. Gray level changes are used to describe the picture corresponding to the derivative of the picture's twodimensional function. The highest difference in gray image value is the local peak value of the first derivative and the zero point of the two order derivative. Contrast enhancement is performed on an infrared picture using the Top and Bottom-Hat transforms of Chiwu Bu et al. [12]:

$$
\begin{aligned}
& \operatorname{HAT}(A)=A-(A \circ B) \\
& H A T^{\prime}=(A \cdot B)-A \\
& f \circ g=(f \Theta g) \oplus g \\
& f \cdot g=-[(-f) \circ(-g)] \\
& \operatorname{HAT}(A)=A-(A \circ B)=A-(A \Theta B) \oplus g \\
& \operatorname{HAT}^{\prime}(A)=(A \cdot B)-A=-A-[(-A) \circ B]=\operatorname{HAT}(-A)
\end{aligned}
$$


Where:

- $\mathrm{A}$ is the infrared image;

- $\quad \mathrm{B}$ is the thermal anomaly element on the infrared image;

- $f$ is the gray value function of pixels $\mathrm{x}$ and $\mathrm{y}$;

- $g$ is the filter function of pixels $\mathrm{x}$ and $\mathrm{y}$;

- $f \circ g$ is the opening operation of the gray value;

- $f \cdot g$ is the closing operation of the gray value;

- $\operatorname{HAT}(A)$ is the Top-Hat transformation;

- $\operatorname{HAT}^{\prime}(A)$ is the Bottom-Hat transformation;

The Top-Hat transform can identify relatively bright things against a dark backdrop, whereas the Bottom-Hat transform can identify somewhat unclear things against a light background. As a result, pixel locations of hot areas in an infrared picture may be recognized using the Top-Hat, and Bottom-Hat transforms [11]. This research looks into this method to localize anomalies and then calculate spatial coordinates of the thermal anomalies. Processing results are shown in Figure 7. Because edge detection is sensitive to picture noise, the first step is to eliminate the picture's noise with a Gaussian filter. Chiwu $\mathrm{Bu}$ et al. [11] use the $5 \times 5$ parameter for the Gaussian filter. This research is checked by using $3 \times 3,5 \times 5$, and $8 \times 8$ parameters for the Gaussian filter. The optimal value between noise and data loss is the $5 \times 5$ parameter. The second step after blurring is to apply the watershed algorithm [13].

The watershed algorithm is a type of picture segmentation method based on morphology theory. Grayscale levels are presented in Figure 7a. The picture following the Top-Hat transform is displayed in Figure 7b, in which brighter items in the black backdrop falling can be measured, and the size can be approximated using the average radius of the measured objects in the picture. The picture following the Bottom-Hat transform is shown in Figure 7c. The merge of Top-Hat and Bottom-Hat creates full anomaly size for opening operation. The result of the merge is presented in Figure $7 \mathrm{~d}$. In the end, it requires applying a grey value opening operation to create a binary bitmap for the subsequent edge detection [14].

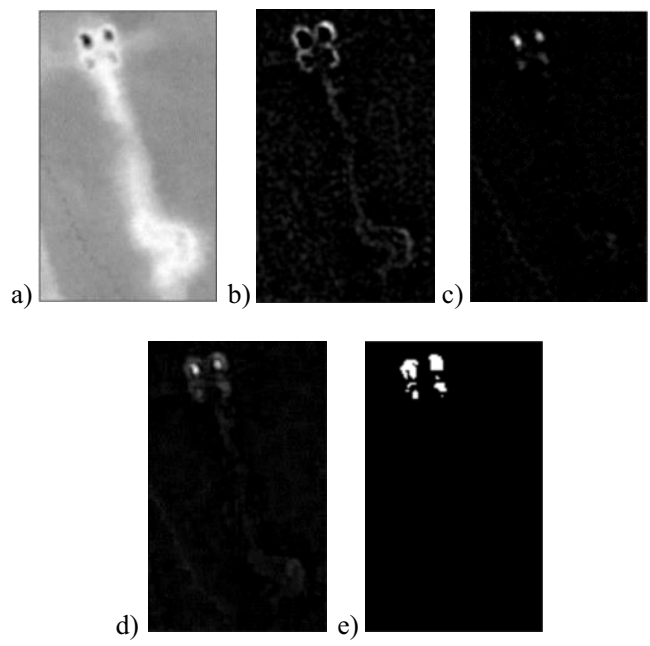

Figure 7. Processing result: a) grayscale change; b) Top-Hat transform; c) Black-Hat transform; c) gray value opening operation; d) Top-Hat transform + Black-Hat transform; e) gray value opening operation; [11]. 


\subsubsection{Object Edge Detection}

The characteristic knowledge of the deficient area is obtained after segmentation of the Watershed. It is then required to classify candidate's edges using a popular edge detection algorithm- the Canny Edge Detection [12], as seen in Figure 8b. As a consequence of the processing step, a significant amount of worthless and untrue boundary data can be minimized, increasing the leakage extraction function effect: Figures $8 \mathrm{a}$ and $8 \mathrm{~b}$ show the approach proposed in this explorative research for filtering relevant data. Figures $8 \mathrm{c}$ and Figure $8 \mathrm{~d}$ show the classical method of filtering.

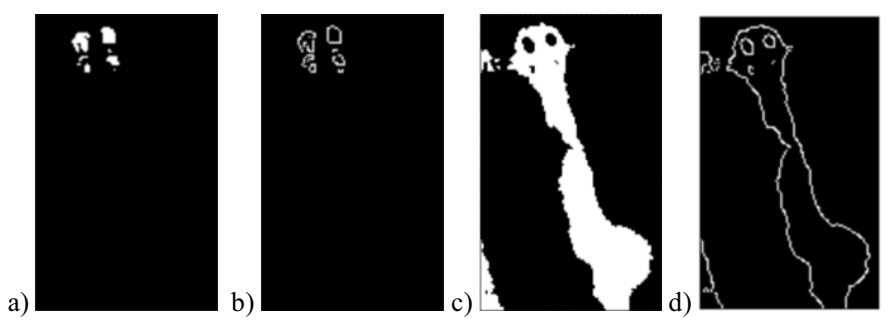

Figure 8. Result of edge detection: a) thermal image with gray value opening operation; b) edge detection by Canny operator in the thermal image with gray value opening operation; c) classic approach of opening operation with basic thresholding; d) edge detection by Canny operator in the thermal image with basic thresholding; [11].

\subsubsection{Leakage Position Extraction}

As a result of this phase, the thermal data is represented as spatial objects. The detected object on the thermal image is assigned to one of three categories: water leakage, heat leakage, or unrelated. The water leakage object means that the $\mathrm{DH}$ network has an emergency. The heat leakage implies that part of the DH network has a thermal insulation issue. This issue is not critical and can be postponed. A not related type of problem means that other reasons cause the thermal anomaly. It is worth noting that some of the intensely glowing objects can also be detected as leaks. As a result of this step, the thermal data is presented in the form of spatial coordinates. Next, it is necessary to calculate the expected place of the leak to carry out a semantic search in the future. It is used the algorithm for finding the center of the blob (centroid). The centroid of the shape is the arithmetic mean of all points in the shape of the figure has $n$ different points with coordinates $x_{1} \ldots x_{n}$ and $y_{1} \ldots y_{n}$, then the centroid $c(x, y)$ is given as

$$
c_{x}=\frac{1}{n} \sum_{i=1}^{n} x_{i}, c_{y}=\frac{1}{n} \sum_{i=1}^{n} y_{i}
$$

Each form in image processing and computer vision is built up of pixels, and the centroid is simply the weighted average of all the pixels that comprise the shape. After the centroid is calculated, most of the incorrectly identified areas are removed. However, some large, unrelated objects can still be preserved as heat anomalies. Spatial Data on the DH network is used for checking the anomaly's intersection with the network to remove heat anomalies not related to the $\mathrm{DH}$ network or permissible heat losses at engineering facilities. An algorithm (Algorithm 1) for eliminating thermal noise from a heat map for an area of underground DH networks is described below. 


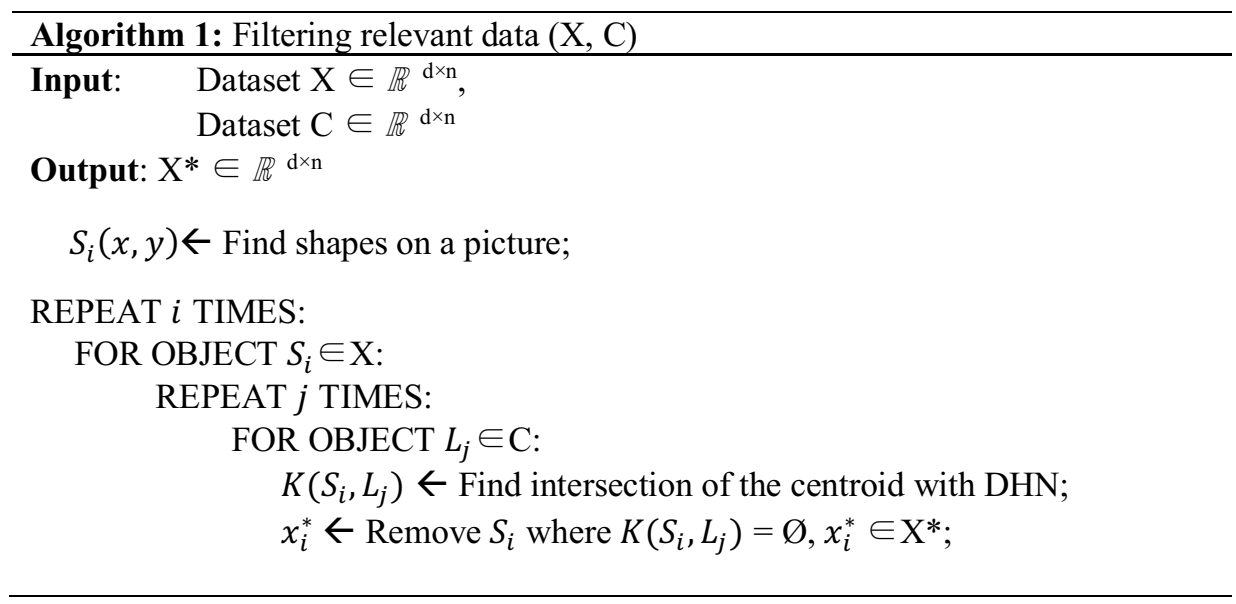

\section{Semantic Space for Calculation}

The research work of S. Sasaki at el. [6] use semantic range as simple fuzzy logic in semantic computation to define a range of semantics according to each standard. Each element of the DH network is assessed according to the range of parameter values [15]. Those values are interpreted as an emergency, problematic and good. For example, if a hole in the pipeline in a network section, the DH network goes into an emergency state. The DH network becomes a problematic state when the DH network doesn't have water leakages but presents thermal insulation problems of heat leaks. If a section or the entire DH network sensor values have acceptable values, such a section or DH network has a good condition. The number of states can be expanded depending on the events needing to be monitored. For the DH network state evaluation method, these influences are defined as the semantics of the DH network state.

The most important feature of the method is that the system provides an interpreter [1] to calculate the quality level and convert influences/meanings into a sentence or a set of words that even non-specialists or ordinary people can understand. Secondly, [2] implement semantic calculations to target the values of multi-parameter sensing. Thus, the semantic space for calculating relationships or correlations between each parameter's values and the semantics is presented in Figure 9.

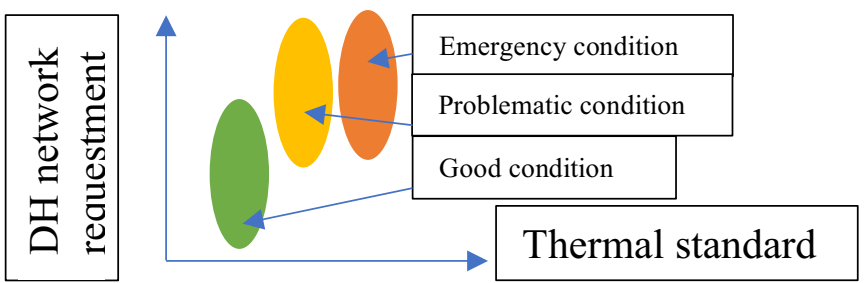

Figure 9. Semantic space for the DH measurement analysis. 


\section{Sensing Measurements Range Calculation and Priority Factor}

In reality, sensing measurement semantics have a higher priority than infrared image semantics. Without emergency readings from the sensors, the system cannot go into an emergency state even if thermal anomalies are present in the infrared images. Therefore, a priority factor $\mathrm{k}$ is introduced to add weight to the calculation. S. Sasaki et al. [6] apply simple Fuzzy logic to defining the range of semantics according to each standard called Semantic Range (SR). This research also uses the same solution. The minimum value of a parameter in measurement is defined as $S R$-min, the maximum value is defined as $S R$ max, and the $S R$-mean is defined as a mean value. For each parameter value, the measured value's total point in the fuzzy-set interval is determined as the sum of each weight [6]. Weight uses 0 value to excluded un-used measurement and 1 to include measurement in the semantic calculation.

Table 2. Definition of fuzzy-set interval and the membership function

\begin{tabular}{rc}
\hline Definition & Weight \\
\hline$S R$-min & 0 \\
$S R$-mean & 1 \\
$S R$-max & 0
\end{tabular}

\section{Conclusion}

This paper describes how the industry can use semantic computing SPA to identify water leaks using multi-parameter sensors and thermal images of the ground surface near DH pipelines to monitor emergencies in the system. The paper explores the integration of SPA functions to analyze multi-dimensional data to create a monitoring system for DH networks. It is assessed that the SPA approach is a suitable candidate to be used for monitoring emergency events in the DH network. It is found that during the research process, several dozen conditions are applied to verify the problem identification conditions. In this case, it is possible to use the knowledge of the correct state as a condition for verification. It would be good to separate the knowledge of the correct condition and observe the situation. Thus, it would give fast state monitoring without changing the correct state knowledge. The main feature of the proposed approach is the integration of sensor data semantics and thermal anomaly search from infrared image databases to identify the leak location. This research aims to implement a search for the coordinates of the problem based on sensor and thermal image data and create a list of problems based on the search results.

\section{References}

[1] Lah AAA, Dziyauddin RA, Md Yusoff N. Localization Techniques For Water Pipeline Leakages: A Review. In: 2018 2nd International Conference on Telematics and Future Generation Networks (TAFGEN) [Internet]. IEEE; 2018. p. 49-54. Available from: https://ieeexplore.ieee.org/document/8580467/

[2] Zhou S, O’Neill Z, O’Neill C. A review of leakage detection methods for district heating networks. Appl Therm Eng [Internet]. 2018 Jun;137:567-74. Available from: https://linkinghub.elsevier.com/retrieve/pii/S1359431118301169

[3] Chan TK, Chin CS, Zhong X. Review of Current Technologies and Proposed Intelligent Methodologies for Water Distributed Network Leakage Detection. IEEE Access [Internet]. 2018;6:78846-67. Available 
from: https://ieeexplore.ieee.org/document/8565861/

[4] Hevner, March, Park, Ram. Design Science in Information Systems Research. MIS Q [Internet]. 2004;28(1):75. Available from: https://www.jstor.org/stable/10.2307/25148625

[5] Kiyoki Y, Chen X, Veesommai C, Rachmawan IE, Chawakitchareon P. A SPA-Based Semantic Computing System for Global \& Environmental Analysis and Visualization with "5-Dimensional WorldMap": “Towards Environmental Artificial Intelligence.” Inf Model Knowl Bases XXXI. 2020;321:285.

[6] Sasaki S, Kiyoki Y. Analytical Visualization Function of 5D World Map System for Multi-Dimensional Sensing Data. Inf Model Knowl Bases. 2018;29:71-89.

[7] Rungsupa S, Chawakitchareon P, Hansuebsai A, Sasaki S, Kiyoki Y. Photographic assessment of coral stress: Effect of low salinity to Acropora sp. Goniopora sp. and Pavona sp. at Sichang Island, Thailand. Inf Model Knowl Bases XXIX. 2018;301:137-48.

[8] Kiyoki Y, Chen X, Veesommai C, Sasaki S, Uraki A, Koopipat C, Chawakitchareon P, Hansuebsai A. An Environmental-Semantic Computing System for Coral-Analysis in Water-Quality and Multi-Spectral Image Spaces with” Multi-Dimensional World Map. Inf Model Knowl Bases XXIX. 2018;301:52-70.

[9] Nemchenko VI, Zheltukhin AA. The System Analysis Of Regulation Of Thermal Loading And Increase Of Efficiency Heat Supply Of Micro-area Of Samara. Constr Archit [Internet]. 2010;(7):172-9. Available from: https://cyberleninka.ru/article/n/sistemnyy-analiz-regulirovaniya-teplovoy-nagruzki-i-povyshenieeffektivnosti-teplosnabzheniya-mikrorayona-g-samary

[10] Kudinov I V., Kolesnikov S V., Eremin A V., Branfileva AN. Computer models of complex multiloop branched pipeline systems. Therm Eng [Internet]. 2013 Nov 11;60(11):835-40. Available from: http://link.springer.com/10.1134/S0040601513080053

[11] Politerm, OOO [Internet]. 2021 [cited 2021 Jul 25]. Available from: https://www.politerm.com/

[12] Bu C, Sun Z, Tang Q, Liu Y, Mei C. Thermography Sequence Processing and Defect Edge Identification of TBC Structure Debonding Defects Detection Using Long-Pulsed Infrared Wave Non-Destructive Testing Technology. Russ J Nondestruct Test [Internet]. 2019 Jan 26;55(1):80-7. Available from: http://link.springer.com/10.1134/S1061830919010030

[13] Moga AN, Gabbouj M. Parallel image component labelling with watershed transformation. IEEE Trans Pattern Anal Mach Intell [Internet]. 1997 May;19(5):441-50. Available from: http://ieeexplore.ieee.org/document/589204/

[14] Peters JF. Foundations of Computer Vision: Computational Geometry, Visual Image Structures and Object Shape Detection. Foundations of Computer Vision. Cham: Springer International Publishing AG; 2017. (Intelligent systems reference library; vol. 124).

[15] Kiyoki Y, Kitagawa T, Hayama T. A metadatabase system for semantic image search by a mathematical model of meaning. ACM SIGMOD Rec [Internet]. 1994 Dec;23(4):34-41. Available from: https://dl.acm.org/doi/10.1145/190627.190639 